\title{
Tecnura
}

http://revistas.udistrital.edu.co/ojs/index.php/Tecnura/issue/view/640

DOI: http://dx.doi.org/10.14483/udistrital.jour.tecnura.2014.SE1.a05

INVESTIGACIÓN

\section{Modelo para identificación de cargas perturbadoras de la calidad de potencia eléctrica en cuanto al fenómeno armónico en una s/e}

\author{
Model for identifying disturbing loads of electric power quality \\ in terms of the harmonic phenomenon in s/e
}

\author{
Julio César Moreno Cañón*, Charles Aguirre Buitrago**, \\ Luis Antonio Noguera Vega***
}

Citation / Para citar este artículo: Moreno Cañón, J. C., Aguirre Buitrago, C., \& Noguera Vega, L. A. (2014). Modelo para identificación de cargas perturbadoras de la calidad de potencia eléctrica en cuanto al fenómeno de armónicos en una S/E. Revista Tecnura, Edición especial, 65-79.

Fecha de recepción: 29 de noviembre de 2013 / Fecha de aceptación: 11 de julio de 2014

\section{RESUMEN}

En este documento se presenta el modelo en particular con el propósito de identificar y enfocar la carga no lineal en el sistema eléctrico de un operador de red. Debido a los nuevos requisitos y parámetros de la regulación, hoy es necesario comenzar a establecer, localizar y desactivar el usuario culpable; de lo contrario, la compañía eléctrica tendrá el deber de garantizar mejor calidad de poder. A través de la estadística, en este caso con ayuda de correlación, se describen los parámetros eléctricos y características de los usuarios.

Palabras clave: Calidad de potencia, cargas no lineales, Comisión de Regulación de Energía y Gas (CREG), correlación, distorsión armónica.

\begin{abstract}
This document presents the particular model with the purpose to identify and focus non-linear load in the electric system of a network operator. Due to the new requirements and parameters of regulation, today it is necessary to begin establishing, locating and deactivating the guilty user, otherwise, the electric company will guarantee better power quality. Through statistics, in this case with correlation support, the electrical parameters and users characteristics are described.
\end{abstract}

Keywords: Correlation, CREG (Colombian Comisión de Regulación de Energía y Gas), harmonic distortion, non-linear loads, quality power.

\footnotetext{
* Ingeniero electricista. Profesional en Distribución de CODENSA S.A ESP. Bogotá, D.C. Contacto: jmorenoca@endesacolombia.com.co ** Ingeniero electricista. Profesional en Distribución de CODENSA S.A ESP. Bogotá, D.C. Contacto: chaguirre@endesacolombia.com.co

*** Ingeniero en Distribución y Redes Eléctricas, especialista en Informática y Automática Industrial. Docente, Universidad Distrital Francisco José de Caldas. Bogotá, D.C. Contacto: lanoguerav@udistrital.edu.co
} 


\section{INTRODUCCIÓN}

Este documento presenta el desarrollo de un modelo para la identificación y focalización de cargas perturbadoras de la calidad de potencia en cuanto al fenómeno de distorsión armónica en subestaciones de $34,5 \mathrm{kV}$ y $11,4 \mathrm{kV}$, buscando satisfacer las necesidades en la calidad de la onda de tensión de usuarios particulares del sistema y dar cumplimiento a lo estipulado en la regulación actual de calidad de potencia en Colombia (Resolución CREG 016 de 2007, por la cual se modifica parcialmente la Resolución CREG 024 de 2005 que establece las normas de calidad de potencia eléctrica aplicables a los servicios de distribución de energía eléctrica) que estipula un plazo máximo de treinta (30) días hábiles para que el operador de red $(\mathrm{OR})$ luego de la detección de una deficiencia en la calidad de la potencia suministrada identifique al usuario causante de la misma. Si vencido este plazo no lo ha identificado, el OR deberá proceder a corregir dicha deficiencia (Comisión de Regulación de Energía y Gas, 2005).

Ahora bien, se efectúa un análisis del marco bibliográfico y regulatorio a nivel mundial con el ánimo de establecer el futuro próximo de la regulación del fenómeno armónico en Colombia, asignando oportunidades de mejora y necesidades próximas en la medición, identificación y control de las perturbaciones del sistema por parte del OR.

De acuerdo con lo anterior, se desarrolla un modelo particular combinando estadística descriptiva con la conexión y desconexión de cargas (por eventos del sistema de distribución local) para focalizar los usuarios más probables de ser fuentes de perturbación armónica.

\section{MARCO CONCEPTUAL Y REGULATORIO}

La conexión de elementos no lineales al sistema eléctrico cambia la naturaleza de la onda sinusoidal de la corriente de alimentación, generando un flujo de corrientes sinusoidales con diferente frecuencia; este fenómeno se conoce como distorsión armónica. En la figura 1 se observa que cuando una forma de onda es idéntica en cada uno de sus ciclos, esta puede representarse como la suma de ondas sinusoidales en las que la frecuencia de cada onda es un múltiplo entero de la frecuencia fundamental de la onda distorsionada; esta suma se conoce como series de Fourier.

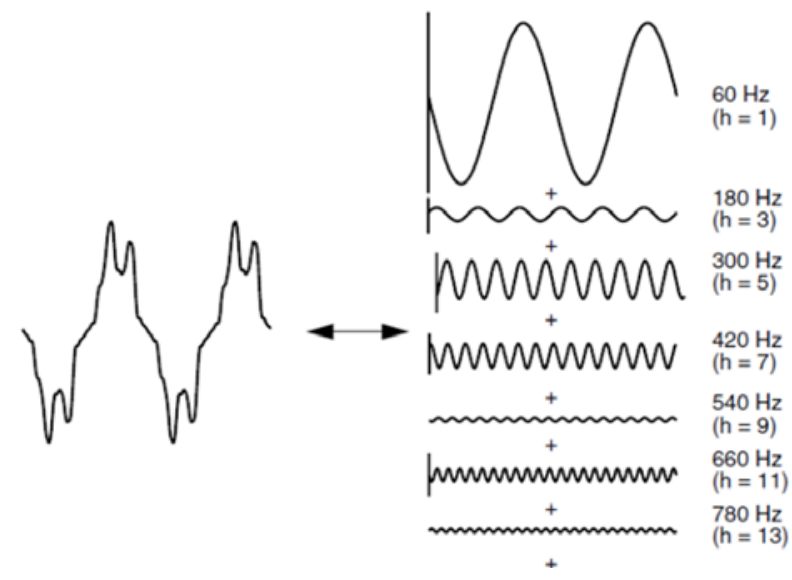

Figura 1. Representación en series de Fourier de una onda distorsionada.

Fuente: (Dugan, Santoso, \& Wayne, 2013).

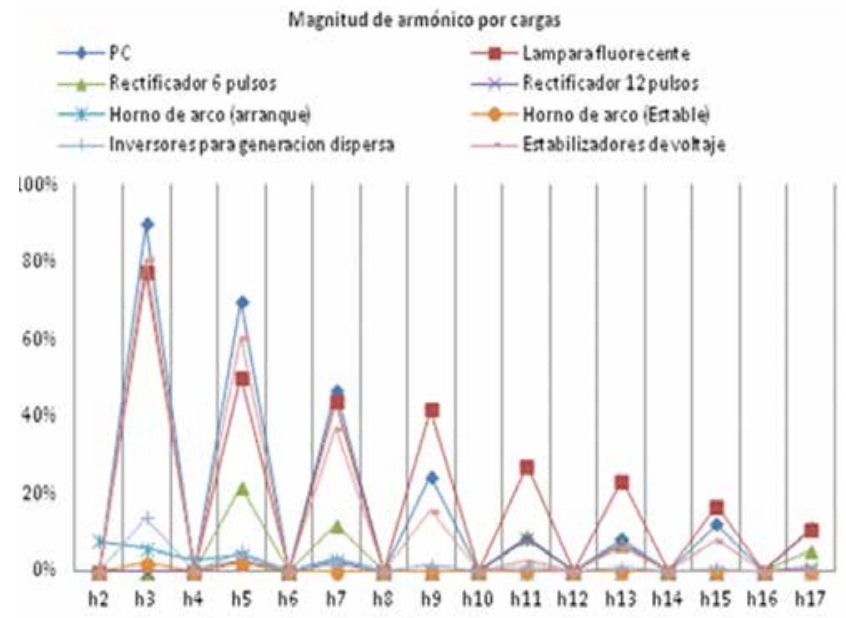

Figura 2. Magnitudes típicas de armónicos en cargas conectadas al sistema eléctrico.

Fuente: Elaboración propia. 
De acuerdo con la aplicación de las cargas no lineales conectadas al sistema, las causas de la generación de corrientes armónicas se pueden separar de acuerdo con el tipo de cliente para este trabajo serán los Ilamados industriales, comerciales y residenciales. En la figura 2 se observa la magnitud de los armónicos en porcentaje de la fundamental en algunas de las cargas más comunes en un sistema de distribución.

Las corrientes armónicas generadas por las cargas no lineales conectadas al sistema pueden impactar negativamente en una gran cantidad de equipos conectados a la red distorsionada, ocasionando pérdidas adicionales, sobrecalentamiento y sobrecargas.

En los transformadores, la distorsión armónica contribuye significativamente al sobrecalentamiento debido al efecto Joule, aumentando las pérdidas en el cobre y en el núcleo (Pereira, 2010). Respecto a los conductores expuestos a distorsión armónica en la corriente transportada, estos se ven expuestos a calentamientos por los efectos conocidos como piel y proximidad, que varían en función de la frecuencia y del calibre del conductor, y como resultado de este par de efectos, la resistencia efectiva de los conductores incrementa aumentando las pérdidas (IEEE, 1992).

Las componentes armónicas afectan los instrumentos de medida ocasionando errores positivos y negativos, dependiendo del tipo de medidor y de los armónicos involucrados. En general, el factor de distorsión debe ser elevado (> 20\%) antes de que se evidencien errores significativos en los aparatos de medida (IEEE, 1992).

La comparación de los límites y controles regulatorios relacionados con la distorsión armónica a nivel mundial nos sitúa en el escenario actual y deja entrever el futuro de la regulación armónica en Colombia. Dicha comparación se logra recopilando la información regulatoria del fenómeno armónico en los países referentes latinoamericanos (Argentina, Brasil y México), además de Estados Unidos, España y Sudáfrica; clasificando sus límites, controles y penalizaciones por los niveles de tensión estipulados por la CREG 082 de 2002 (tabla 1), se encontraron diferencias entre los países en cuanto a la aplicación de penalizaciones a los OR, el control de las emisiones a los usuarios finales en el punto de conexión común (PCC) y los límites establecidos para la distorsión armónica de las ondas de tensión y corriente (Pereira, 2010).

Tabla 1. Niveles de tensión.

\begin{tabular}{cl}
\hline $\begin{array}{c}\text { Nivel de } \\
\text { tensión }\end{array}$ & \multicolumn{1}{c}{ Rango de tensión } \\
\hline I & Menor que $1[\mathrm{kV}]$ \\
\hline II & Mayor o igual a $1[\mathrm{kV}]$ y menor a $30[\mathrm{kV}]$ \\
\hline III & Mayor o igual a $30[\mathrm{kV}]$ y menor a $57,5[\mathrm{kV}]$ \\
\hline IV & Mayor o igual a $57,5[\mathrm{kV}]$ y menor a $220[\mathrm{kV}]$ \\
\hline
\end{tabular}

Fuente: Elaboración propia.

La regulación en Colombia es la única que no limita los valores individuales de distorsión armónica de tensión. Al analizar en detalle los límites regulatorios se encuentran diferentes exigencias en los países, en cuanto a la distorsión armónica total de tensión, como se puede observar en la figura 3, España no la limita para los niveles de tensión más altos, además de que los límites más exigentes son los estadounidenses junto con los colombianos (basados en la IEEE 519) y dejando los límites regulatorios de Brasil como los menos estrictos en cuanto al THDv.

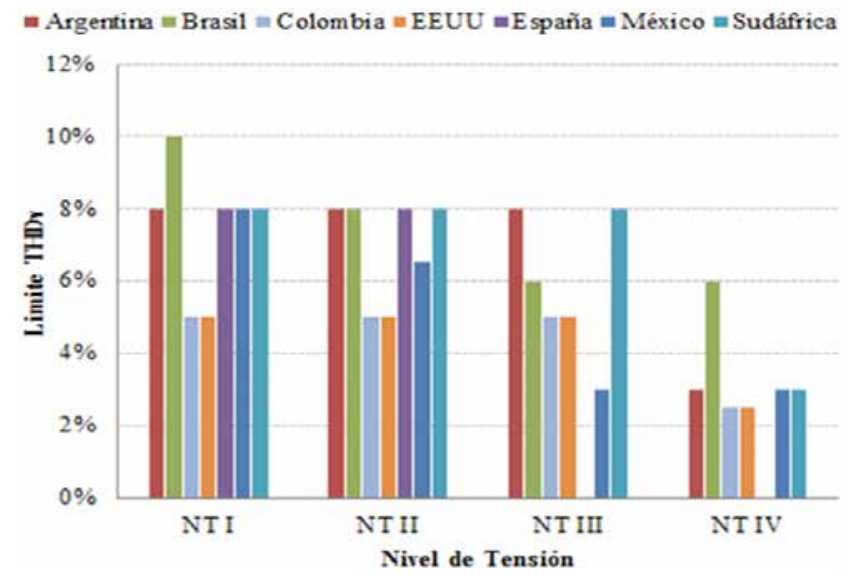

Figura 3. Límites regulatorios para la distorsión armónica total de tensión (THDv).

Fuente: Elaboración propia. 
Para los límites individuales de distorsión armónica en la onda de tensión (no regulados en Colombia, figura 4), se encuentra que los límites más exigentes son para los armónicos pares y los múltiplos de tres y que dichos límites se tornan más estrictos para los niveles de tensión más altos, situación que pone en desventaja el actual marco regulatorio de calidad de potencia en Colombia, el cual solo limita la distorsión armónica total de tensión (THDv).

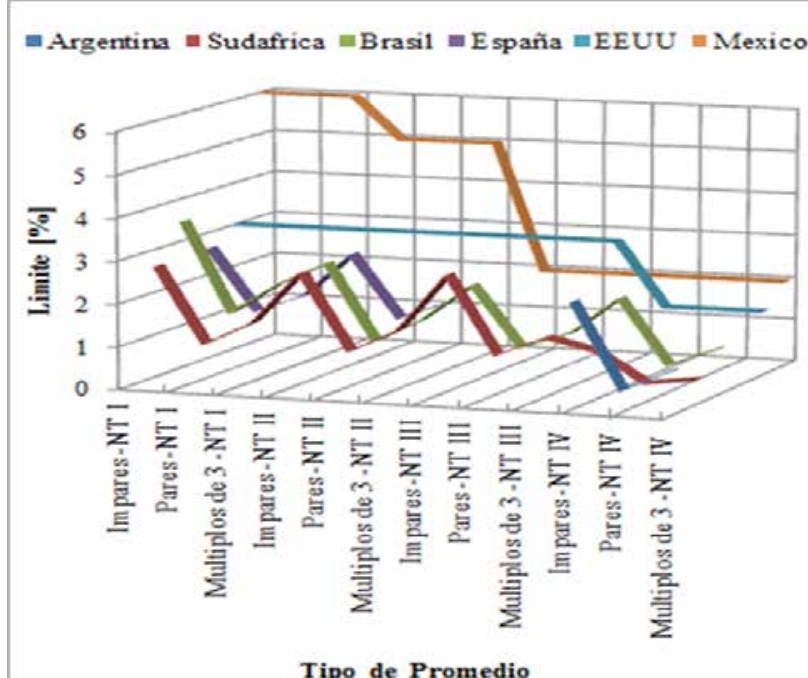

Figura 4. Límites regulatorios para la distorsión armónica individual de tensión (IHDv).

Fuente: Elaboración propia.

\section{METODOLOGÍA}

De acuerdo con el desafío planteado en la regulación colombiana, luego de identificada una perturbación en la calidad de la potencia superior a los límites determinados se requiere la identificación por parte del OR, del usuario causante de la misma en un plazo no mayor que treinta (30) días hábiles para evitar asumir por completo la corrección de dicha deficiencia (Comisión de Regulación de Energía y Gas, 2007) a fin de garantizar el cumplimiento regulatorio, cuando se detecta una distorsión armónica de la onda de tensión superior a 5\% (niveles de tensión I, II y III), se desarrolló un modelo que inicialmente tipifica la carga total conectada a cada barraje y la compara por medio del coeficiente de correlación momento producto de Pearson $(r)$ con la caracterización por estadística descriptiva de la distorsión armónica presente en cada barra, determinando así el comportamiento característico de la distorsión armónica en las redes respecto a los tipos de usuarios que la generan.

Luego son seleccionadas por parte del OR las barras críticas que requieren la aplicación del modelo y en estas se evalúa su comportamiento durante períodos de desconexión y conexión de cargas durante los diferentes eventos del sistema (maniobras de mantenimiento y fallas). El flujograma del modelo propuesto se observa en la figura 5 .

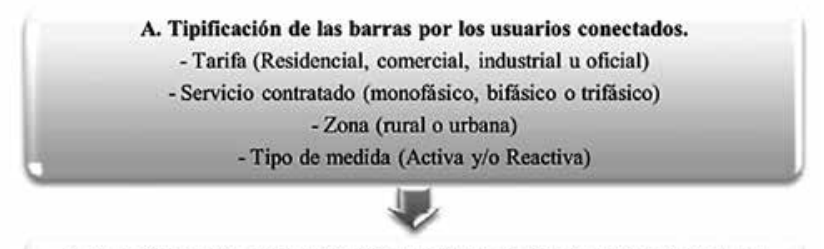

B. Caracterización de la distorsión armónica de las barras de NT II y III. - Distorsión armónica total de tensión y corriente - Distorsión armónica individual de tensión y corriente - Distorsión armonica en periodos especificos de tiempo.

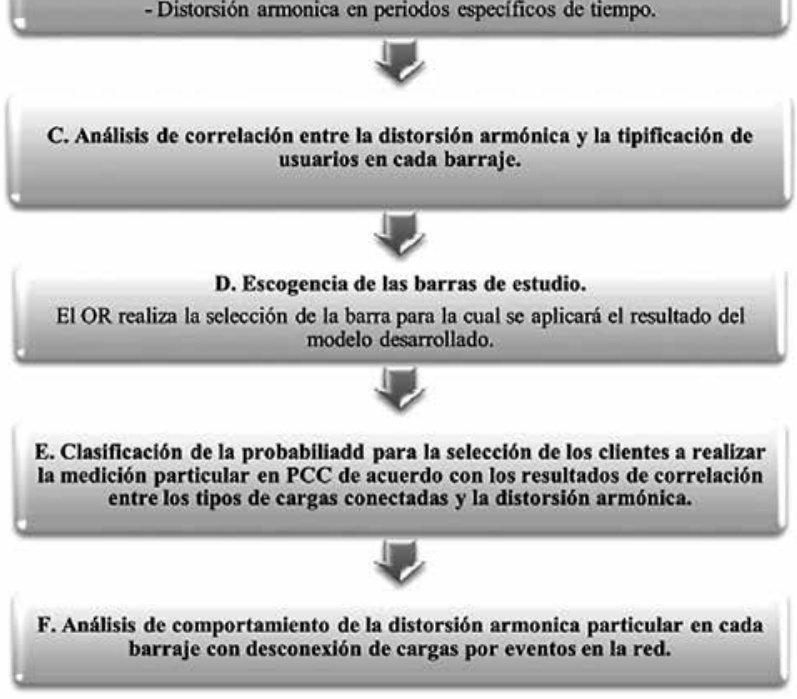

Figura 5. Flujograma para el desarrollo del modelo propuesto.

Fuente: Elaboración propia. 


\section{RESULTADOS}

Después de definir la metodología se procedió a desarrollar cada uno de los pasos definidos, en los cuales se analizó la información del OR.

\section{Tipificación de las barras por los usuarios conectados}

El OR cuenta con una cantidad considerable de subestaciones AT-MT y MT-MT con tensiones de 34,5, 13,2 y 11,4 kV, de donde se alimentan más de 1 millón de usuarios; dichos usuarios se tipificaron por tarifa (residencial, comercial, industrial y oficial, que se entenderá como tipo de carga), servicio contratado (monofásico, bifásico y trifásico), zona (urbana y rural), tipo de medida (activa y/o reactiva) y nivel de tensión en PCC (NT I y NT II y III), siendo el insumo de esta información las bases de datos comerciales de la compañía; esto permite caracterizar las cargas conectadas en cada barraje.

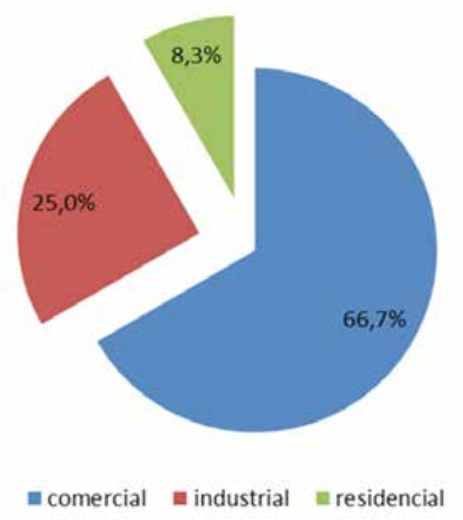

Figura 6. Tipificación de clientes por tarifa en la barra 1 de $34.5 \mathrm{kV}$ de la S/E.

Fuente: Elaboración propia.

Siguiendo las recomendaciones de la NTC 2859 se determina el tamaño de la muestra con el máximo nivel de inspección, resultando una muestra de 50 barrajes, con un nivel de confianza de 91,47\% y un margen de error de $7 \%$, para los cuales se tipificaron sus usuarios (ICONTEC, 2002); en la figura
6 se observa la tipificación de clientes en cuanto a la tarifa para una de las barras seleccionadas por muestreo; esta gráfica solo corresponde a una de las variables nombradas en el párrafo anterior.

\section{Caracterización de la distorsión armónica en los barrajes}

Se realiza la descarga de información desde la plataforma corporativa de calidad de potencia para los barrajes seleccionados en un período de tiempo específico con intervalos de medición de 10 minutos para los siguientes parámetros:

Distorsión armónica individual de corriente del armónico 2 al 6 por fase (IHDc).

- Distorsión armónica individual de voltaje del armónico 2 al 8 por fase. (IHDv).

- Distorsión armónica total de voltaje y corriente por fase. (THDv y THDc).

Luego se hace la segregación de los datos obtenidos por rangos de tiempo semanales (lunes a sábado y domingo) y diarios (día y noche) y se aplica estadística para describir, explorar y comparar los datos a través de medidas de tendencia central y variación (promedio, media, moda, desviación estándar, cuartiles, máximos y mínimos), logrando así la caracterización de la distorsión armónica en cada una de las cincuenta (50) barras muestreadas.

\section{Análisis de correlación entre la distorsión armónica y la tipificación de usuarios en cada barraje}

Se compila la información de la caracterización de los barrajes por los usuarios conectados y la distorsión armónica, resultando una matriz aproximada de 50 filas (cantidad de barrajes muestreados) $x$ 161.500 columnas (características de la carga y la distorsión) para encontrar alguna relación entre las cargas conectadas y se utiliza el coeficiente de correlación producto momento de Pearson ( $r$ ) (Triola, 2009) para medir la fuerza de la relación lineal 
entre los valores cuantitativos apareados $x$ (características de las cargas conectadas) y (características de la distorsión armónica) en la muestra.

Mediante una herramienta computacional se calcula la correlación entre las características de la carga conectada y la distorsión armónica y se concluye que existe correlación lineal significativa, donde se cumple lo siguiente:
- El valor absoluto de $r$ excede 0,361 (Triola, 2009).

- El nivel de significancia sea inferior a 0,01 [lbid.]

Como representación gráfica se realizaron espectros de distorsión para las correlaciones encontradas; en la figura 7 se observa uno de ellos.

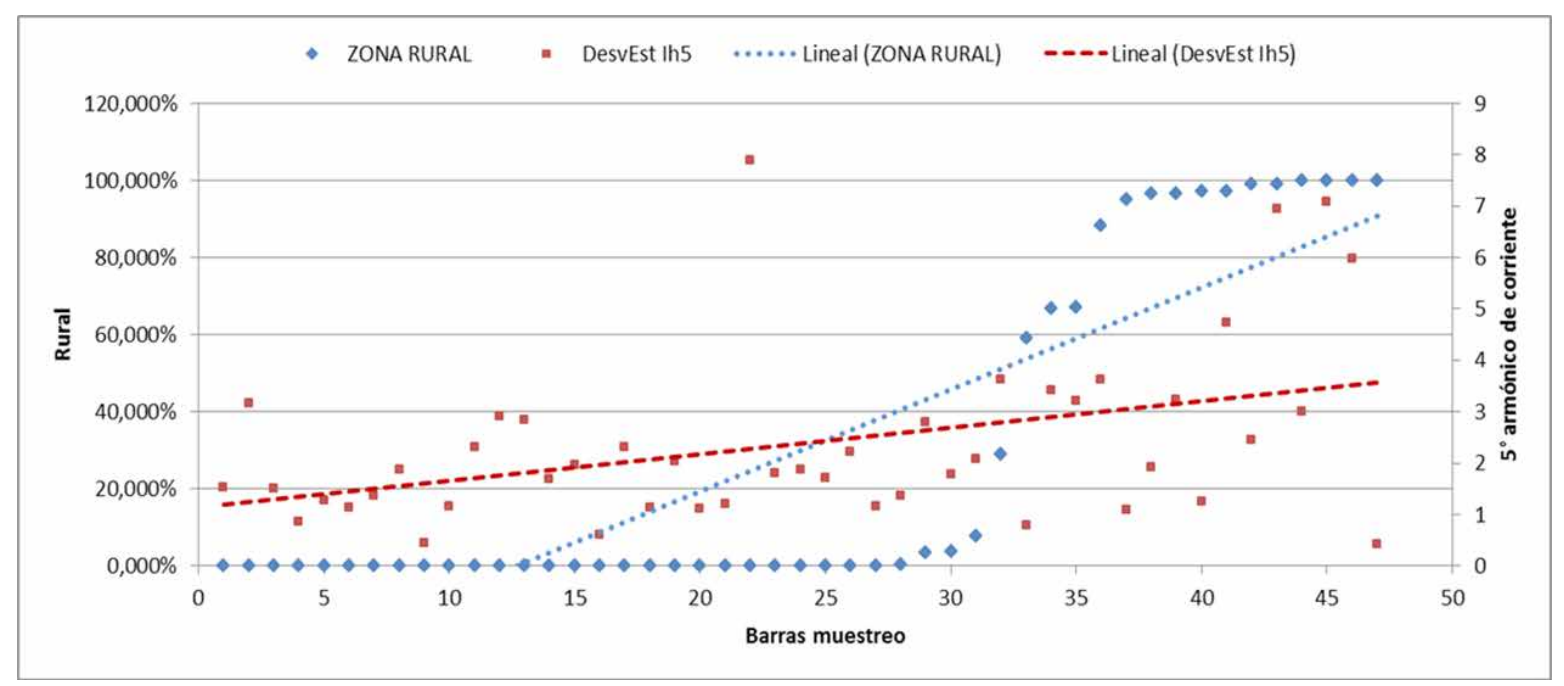

Figura 7. Gráfica de dispersión para la combinación Zona (rural) - Promedio del armónico 5 de corriente.

Fuente: Elaboración propia.

Tabla 3. Resumen de correlaciones entre las características de los clientes conectados y la distorsión de las barras.

\begin{tabular}{|c|c|c|c|c|c|c|c|c|}
\hline Característica & $r(+)$ & r (-) & Min & Max & R1 & R2 & R3 & R4 \\
\hline \multirow{2}{*}{$\%$ IHDv_7 en THDv } & \multirow{2}{*}{$1 \varphi$} & \multirow{2}{*}{$3 \varphi$} & \multirow{2}{*}{$0 \%$} & \multirow{2}{*}{$100 \%$} & $\geq 0[\%]$ & $>25[\%]$ & $>50[\%]$ & $>75[\%]$ \\
\hline & & & & & $\leq 25[\%]$ & $\leq 50[\%]$ & $\leq 75[\%]$ & $\leq 100[\%]$ \\
\hline \multirow{2}{*}{$\begin{array}{l}\text { Dif_IHDi_5 L-S } \\
\text { y Dom (día) }\end{array}$} & \multirow{2}{*}{$3 \varphi$} & \multirow{2}{*}{$1 \varphi$} & \multirow{2}{*}{$0 \%$} & \multirow{2}{*}{$3 \%$} & $\leq 0,75[\%]$ & $>0,75[\%]$ & $>1,48[\%]$ & $>2,22[\%]$ \\
\hline & & & & & & $\leq 1,48[\%]$ & $\leq 2,22[\%]$ & \\
\hline \multirow{2}{*}{ Prom_V_h5 } & \multirow{2}{*}{ Res } & \multirow{2}{*}{ Ind } & \multirow{2}{*}{$0 \%$} & \multirow{2}{*}{$4 \%$} & $\leq 1,01[\%]$ & $>1,01[\%]$ & $>2,02[\%]$ & $>3,02[\%]$ \\
\hline & & & & & & $\leq 2,02[\%]$ & $\leq 3,02[\%]$ & \\
\hline \multirow{2}{*}{ DesvEst_IHDv_7 } & \multirow{2}{*}{ Rur } & \multirow{2}{*}{ Urb } & \multirow{2}{*}{0,00} & \multirow{2}{*}{0,69} & $\leq 0,17$ & $>0,17$ & $>0,35$ & $>0,52$ \\
\hline & & & & & & $\leq 0,35$ & $\leq 0,52$ & \\
\hline \multirow{2}{*}{ \% IHDi_3 en THDi } & \multirow{2}{*}{ Rur } & \multirow{2}{*}{ Urb } & \multirow{2}{*}{$0 \%$} & \multirow{2}{*}{$100 \%$} & $\geq 0[\%]$ & $>25[\%]$ & $>50[\%]$ & $>75[\%]$ \\
\hline & & & & & $\leq 25[\%]$ & $\leq 50[\%]$ & $\leq 75[\%]$ & $\leq 100[\%]$ \\
\hline \multirow{2}{*}{ \% IHDi_5 en THDi } & \multirow{2}{*}{ Urb } & \multirow{2}{*}{ Rur } & \multirow{2}{*}{$0 \%$} & \multirow{2}{*}{$100 \%$} & $\geq 0[\%]$ & $>25[\%]$ & $>50[\%]$ & $>75[\%]$ \\
\hline & & & & & $\leq 25[\%]$ & $\leq 50[\%]$ & $\leq 75[\%]$ & $\leq 100[\%]$ \\
\hline
\end{tabular}

Fuente: Elaboración propia. 
De acuerdo con los resultados obtenidos del análisis de correlación $(r)$, se construyeron las tablas 3 y 4 , en las que se hace una clasificación de probabilidad a los diferentes tipos de clientes de acuerdo con la caracterización de la distorsión armónica y así poder focalizar la medida en el PCC, disminuyendo la población y permitiendo determinar de manera más eficiente la carga perturbadora.

Tabla 4. Clasificación de la probabilidad para selección de clientes.

\begin{tabular}{lll}
\hline \multicolumn{1}{c}{ Rango } & \multicolumn{1}{c}{$\begin{array}{c}\text { Clasificación } \\
\mathbf{r ( + )}\end{array}$} & $\begin{array}{c}\text { Clasificación } \\
\mathbf{r ( - )}\end{array}$ \\
\hline R1: Menor o igual al 25\% & Baja (1) & Alta (4) \\
\hline R2: Entre 25 y $50 \%$ & Media baja (2) & Media alta (3) \\
\hline R3: Entre 50 y $75 \%$ & Media alta (3) & Media baja (2) \\
\hline R4: Mayor que $75 \%$ & Alta (4) & Baja (1) \\
\hline
\end{tabular}

Fuente: Elaboración propia.

Tabla 5. Caracterización de cargas en barras definidas.

\begin{tabular}{|c|c|c|c|c|}
\hline \multirow{2}{*}{\multicolumn{2}{|c|}{$\begin{array}{c}\text { Característica } \\
\text { B12 } \\
\end{array}$}} & \multicolumn{3}{|c|}{ Barra } \\
\hline & & \multirow{2}{*}{$\begin{array}{r}\text { B1 } \\
S / E 1\end{array}$} & B2 & \multirow[b]{2}{*}{ S/E2 } \\
\hline$S / E$ & & & S/E2 & \\
\hline NT [kV] & & 34,5 & 13,2 & 13,2 \\
\hline Circuitos al & imentados & 3 & 2 & 1 \\
\hline Clientes OR & & 107 & 2211 & 4097 \\
\hline \multirow{4}{*}{ Tarifa } & Comercial & $98,13 \%$ & $1,04 \%$ & $2,56 \%$ \\
\hline & Industrial & $1,87 \%$ & $0,05 \%$ & $0,20 \%$ \\
\hline & Oficial & $0,00 \%$ & $0,05 \%$ & $0,59 \%$ \\
\hline & Residencial & $0,00 \%$ & $98,87 \%$ & $96,66 \%$ \\
\hline \multirow{4}{*}{$\begin{array}{l}\text { Servicio } \\
\text { Contratado }\end{array}$} & Monofásico & $0,00 \%$ & $0,14 \%$ & $0,20 \%$ \\
\hline & Bifásico & $0,00 \%$ & $90,28 \%$ & $89,63 \%$ \\
\hline & Trifásico & $3,74 \%$ & $5,92 \%$ & $6,08 \%$ \\
\hline & Sin Información & $96,26 \%$ & $3,66 \%$ & $4,10 \%$ \\
\hline \multirow{2}{*}{ Zona } & Urbano & $10000 \%$ & $2,76 \%$ & $40,86 \%$ \\
\hline & Rural & $0,00 \%$ & $97,24 \%$ & $59,14 \%$ \\
\hline \multirow{2}{*}{$\begin{array}{l}\text { Tipo de } \\
\text { Medida }\end{array}$} & Activa & $97,20 \%$ & $100,00 \%$ & $100,00 \%$ \\
\hline & Activa y Reactiva & $2,80 \%$ & $0,00 \%$ & $0,00 \%$ \\
\hline \multirow{2}{*}{$\begin{array}{l}\text { Nivel de } \\
\text { Tensión }\end{array}$} & NT I & $91,59 \%$ & $100,00 \%$ & $100,00 \%$ \\
\hline & NT II ó NT III & $8,41 \%$ & $0,00 \%$ & $0,00 \%$ \\
\hline
\end{tabular}

Fuente: Elaboración propia.

\section{Selección del barraje}

De acuerdo con las necesidades del OR, se determinan como barras de estudio las siguientes:

- Barra 12 de 34,5 [kV] de la S/E1

- Barras 1 y 2 de 13,2 [kV] de la S/E2

Para las barras seleccionadas se hace la tipificación de los usuarios conectados, así como la caracterización de su distorsión armónica; este resultado se observa en las tablas 5 y 6 .

Tabla 6. Caracterización de la distorsión armónica en barras definidas.

\begin{tabular}{llll}
\hline \multirow{2}{*}{ Característica } & \multicolumn{3}{c}{ Barra } \\
\cline { 2 - 4 } & B12 & B1 & \multicolumn{1}{c}{ B2 } \\
\hline Percentil 95 del THDi & $8,85 \%$ & $22,54 \%$ & $12,72 \%$ \\
\hline Percentil 95 del THDv & $3,28 \%$ & $5,44 \%$ & $4,53 \%$ \\
\hline Percentil 95 del IHDi_3 & $0,66 \%$ & $5,97 \%$ & $11,48 \%$ \\
\hline Percentil 95 del IHDi_5 & $8,61 \%$ & $19,68 \%$ & $5,13 \%$ \\
\hline Percentil 95 del IHDv_3 & $0,29 \%$ & $0,91 \%$ & $0,98 \%$ \\
\hline Percentil 95 del IHDv_5 & $3,25 \%$ & $4,75 \%$ & $3,98 \%$ \\
\hline Percentil 95 del IHDv_7 & $0,68 \%$ & $2,76 \%$ & $2,31 \%$ \\
\hline$\%$ de IHDi_3 en THDi & $4,40 \%$ & $29,64 \%$ & $78,42 \%$ \\
\hline$\%$ de IHDi_5 en THDi & $98,18 \%$ & $89,62 \%$ & $44,28 \%$ \\
\hline Dif_Prom_IHDi_5 L-S & $2,07 \%$ & $0,04 \%$ & $0,35 \%$ \\
y Dom (Día) & $2,55 \%$ & $3,52 \%$ & $2,88 \%$ \\
\hline Prom_IHDv_5 & $13,54 \%$ & $29,74 \%$ & $34,44 \%$ \\
\hline$\%$ de IHDv_7 en THDv & 0,18 & 0,66 & 0,59 \\
\hline DesvEst_IHDv_7 & & &
\end{tabular}

Fuente: Elaboración propia.

Como parte del análisis se efectuó el estudio de los parámetros eléctricos asociados a los barrajes de la subestación para los cuales se tuvo en cuenta el perfil de carga a partir de lo que se catalogó como una semana típica, es decir, el comportamiento promedio del barraje en condiciones normales de servicio; de esta manera se tiene una comparación entre los valores de carga y el factor de armónicos que contienen; para ello se observa el comportamiento del THDv, THDi y los individuales de corriente para el barraje de la subestación S/E1 (figuras 8 y 9). 


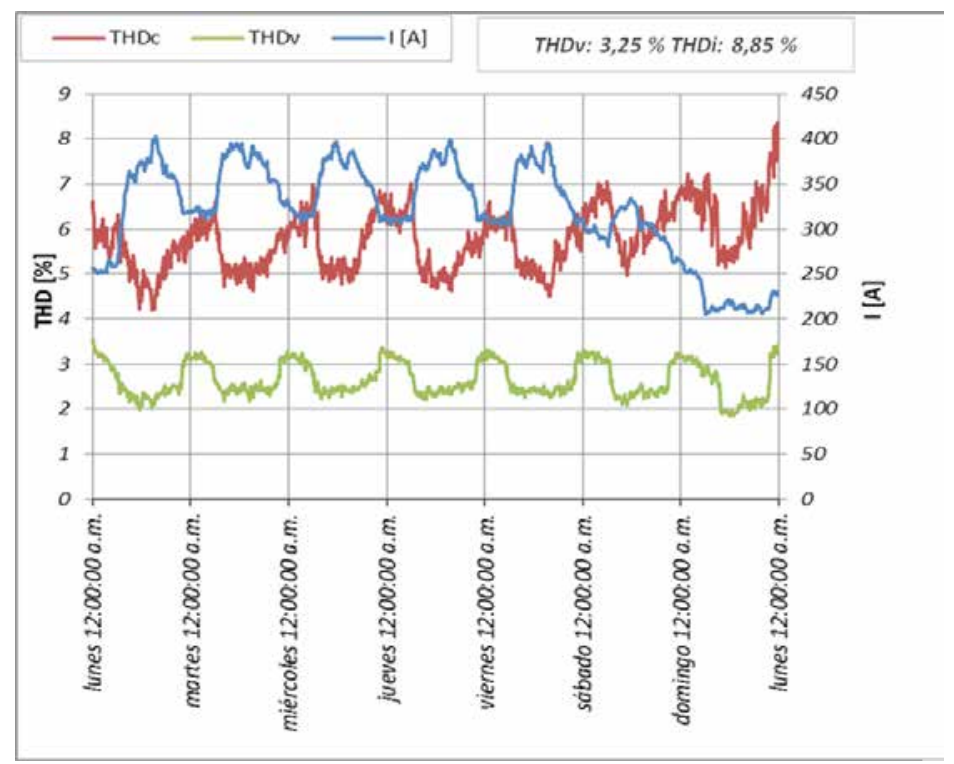

Figura 8. Perfil de carga y distorsión armónica total de tensión y corriente en la barra 12 de 34,5 kV de la S/E1 (semana típica).

Fuente: Elaboración propia.

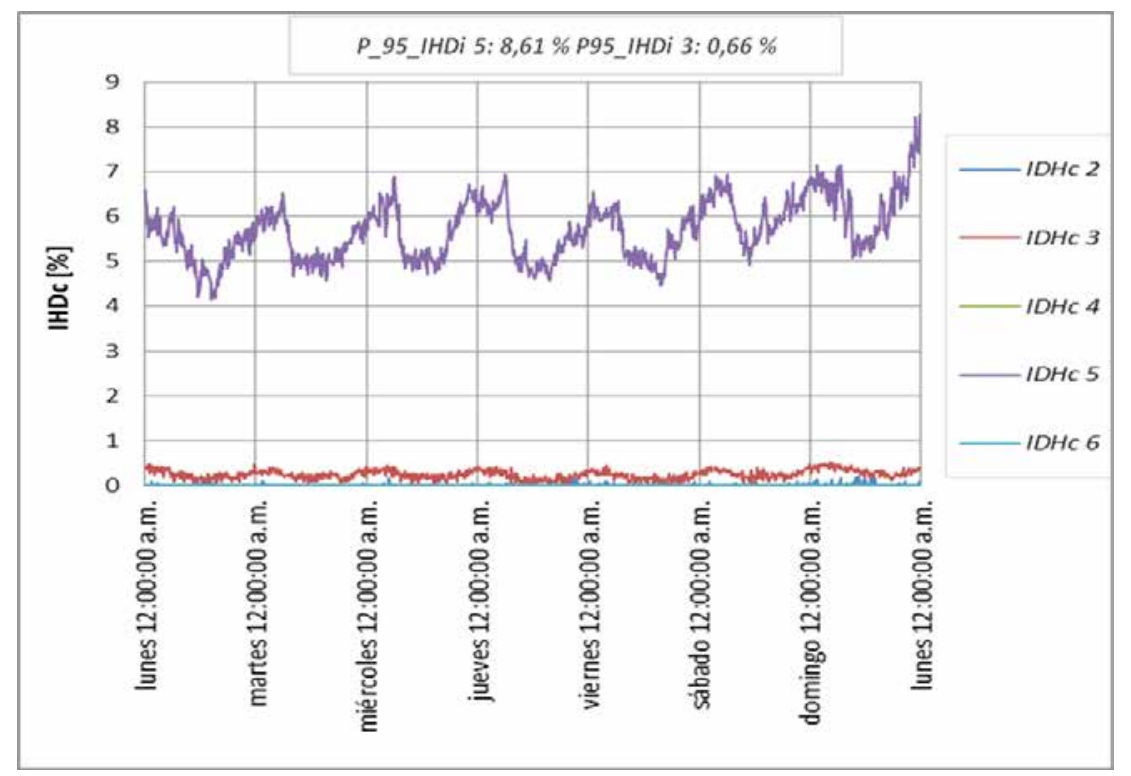

Figura 9. Distorsión individual de corriente en la barra 12 de 34,5 kV de la S/E1 (semana típica).

Fuente: Elaboración propia.

Por otro lado, se tienen los mismos criterios para la subestación S/E2 (la cual tiene dos barrajes); para el caso práctico solo se muestra el comportamiento del barraje 1, en las figuras 10, 11 y 12. 


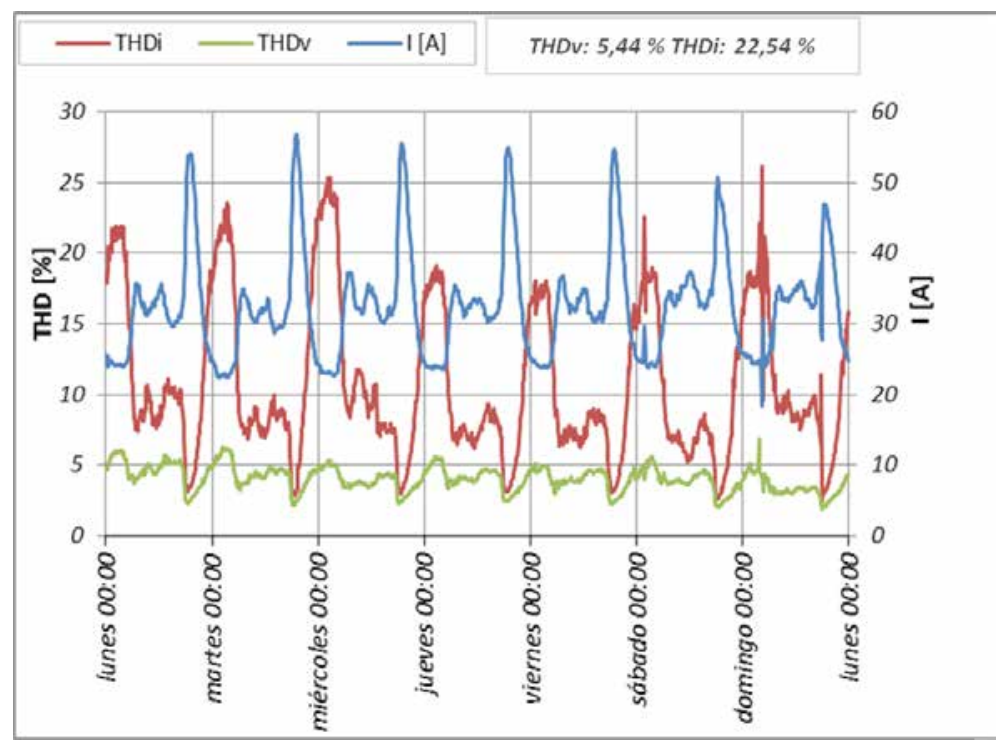

Figura 10. Perfil de carga y distorsión armónica total de tensión y corriente en la barra 1 de 13,2 kV de la S/E2 (semana típica).

Fuente: Elaboración propia,

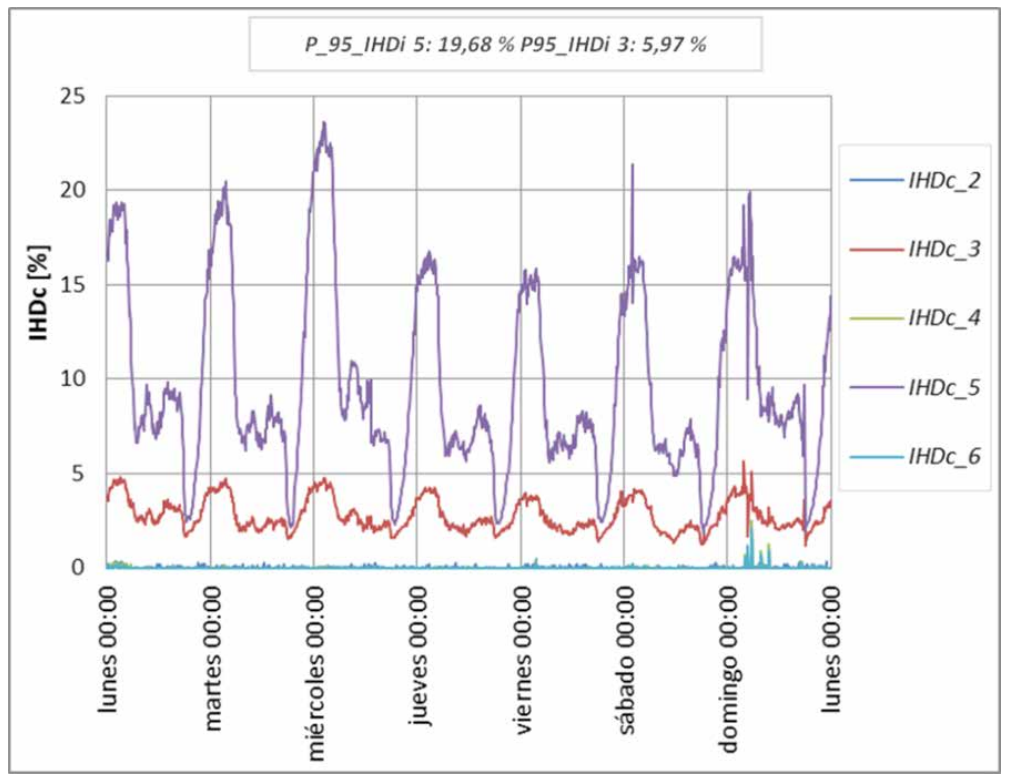

Figura 11. Distorsión individual de corriente en la barra 1 de 13,2 kV de la S/E2 (semana típica).

Fuente: Elaboración propia. 


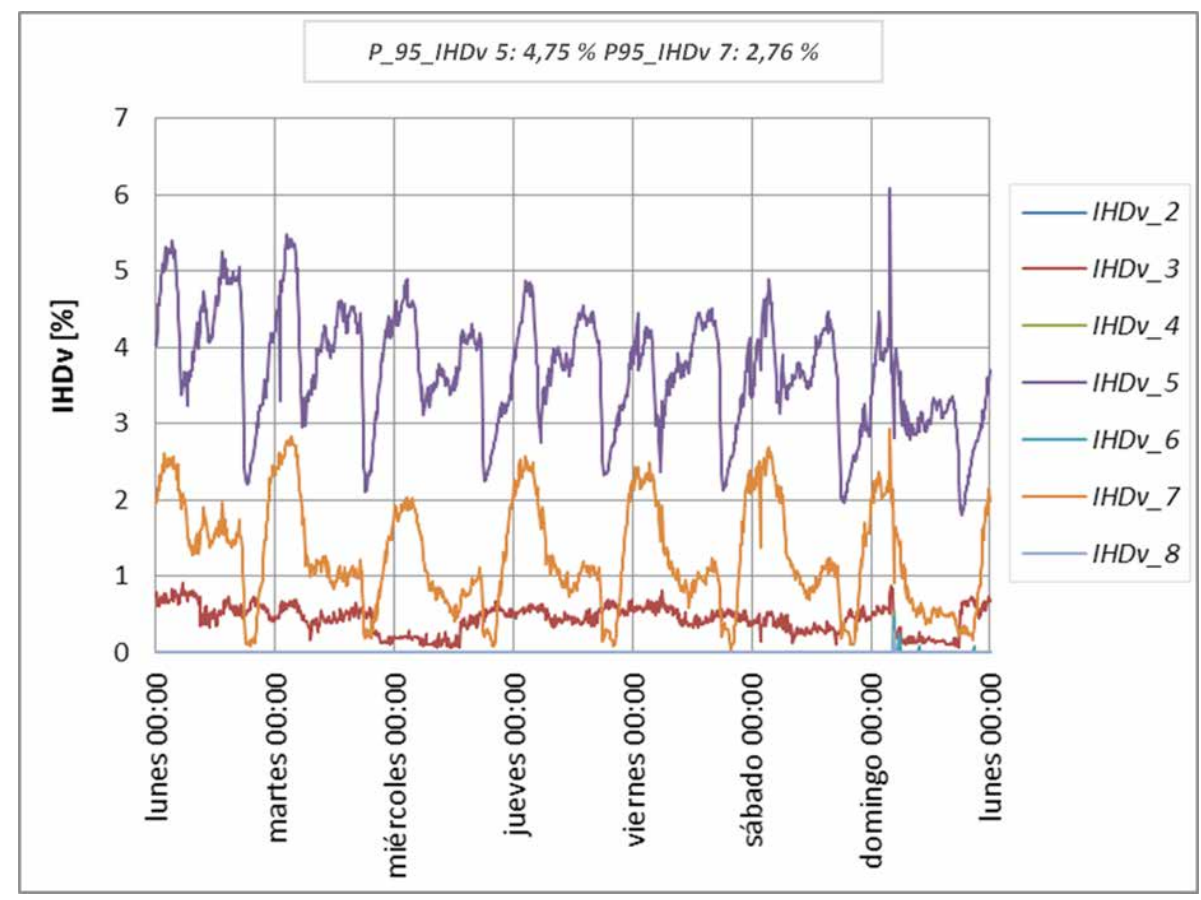

Figura 12. Distorsión individual de tensión en la barra 1 de 13,2 kV de la S/E2 (semana típica).

Fuente: Elaboración propia.

\section{Asignación de rango y clasificación de probabilidades}

Como resultado de la aplicación de las tablas 3 y 4 a la caracterización armónica de cada una de las barras estudiadas, se encuentra que los clientes más probables de generar la distorsión armónica en la barra 12 de $34,5 \mathrm{kV}$ de la subestación S/E1 son los clientes trifásicos, residenciales y urbanos (tabla 7); asimismo para la barra 1 de 13,2 kV de la subestación S/E2 los clientes con mayor probabilidad de ser fuentes de distorsión armónica son los clientes monofásicos, residenciales y ubicados en la zona urbana (tabla 8). Por último, en la barra 2 de 13,2 kV de la subestación S/E2 es preciso iniciar la búsqueda por los clientes monofásicos, residenciales ubicados en la zona rural, como se observa en la tabla 9.

\section{Análisis de eventos con afectación de cargas}

De acuerdo con el modelo planteado, se complementa la priorización con un análisis del comportamiento de la distorsión armónica durante eventos en la red que por su naturaleza llevan a la desconexión y conexión de cargas (fallas y maniobras de mantenimiento) en las barras seleccionadas. Dichos eventos se dividen a su vez en etapas durante las cuales se realiza aperturas y cierres en diferentes dispositivos de maniobra que modifican las cargas afectadas.

Para efectuar este análisis se comparó el comportamiento histórico de la distorsión armónica en cada barra en los períodos de falla y el comportamiento particular en el período de tiempo en que ocurre el evento, dando como resultado cuáles son las cargas que al ser desconectadas del sistema disminuyen en mayor porcentaje la distorsión armónica. 
Tabla 7. Clasificación de la probabilidad para selección de clientes en barraje 12 de 34.5 kV de la subestación S/E1.

\begin{tabular}{|c|c|c|c|c|c|c|c|c|}
\hline \multirow{2}{*}{ Característica } & \multirow{2}{*}{ Valor } & \multirow{2}{*}{ Rango } & \multicolumn{2}{|c|}{ Serv_Contratado } & \multicolumn{2}{|c|}{ Tarifa } & \multicolumn{2}{|c|}{ Zona } \\
\hline & & & $1 \phi$ & $3 \phi$ & Res & Ind & Rural & Urb \\
\hline$\%$ IHDv_7 en THDv & $13,54 \%$ & R1 & Baja (1) & Alta (4) & & & & \\
\hline Dif_IHDc_5 L-S y Dom (día) & $2,07 \%$ & R3 & $\begin{array}{c}\text { Media } \\
\text { Baja (2) }\end{array}$ & $\begin{array}{c}\text { Media } \\
\text { Alta (3) }\end{array}$ & & & & \\
\hline Prom_IHDv_5 & $2,55 \%$ & R3 & & & $\begin{array}{c}\text { Media } \\
\text { Alta (3) }\end{array}$ & $\begin{array}{c}\text { Media } \\
\text { Baja (2) }\end{array}$ & & \\
\hline DesvEst_IHDv_7 & 0,18 & $\mathrm{R} 2$ & & & & & $\begin{array}{c}\text { Media } \\
\text { Baja (2) }\end{array}$ & $\begin{array}{c}\text { Media } \\
\text { Alta (3) }\end{array}$ \\
\hline \% IHDc_3 en THDc & $4,40 \%$ & R1 & & & & & Baja (1) & Alta (4) \\
\hline \% IHDc_5 en THDc & $98,18 \%$ & R4 & & & & & Baja (1) & Alta (4) \\
\hline Total & & & 3 & 7 & 3 & 2 & 4 & 11 \\
\hline
\end{tabular}

Fuente: Elaboración propia.

Tabla 8. Clasificación de la probabilidad para selección de clientes en barraje 1 de 13.2 kV de la S/E2.

\begin{tabular}{|c|c|c|c|c|c|c|c|c|}
\hline \multirow{2}{*}{ Característica } & \multirow{2}{*}{ Valor } & \multirow{2}{*}{ Rango } & \multicolumn{2}{|c|}{ Serv_Contratado } & \multicolumn{2}{|c|}{ Tarifa } & \multicolumn{2}{|c|}{ Zona } \\
\hline & & & $1 \phi$ & $3 \phi$ & Res & Ind & Rural & Urb \\
\hline \% IHDv_7 en THDv & $29,74 \%$ & R2 & $\begin{array}{l}\text { Media } \\
\text { Baja (2) }\end{array}$ & $\begin{array}{l}\text { Media } \\
\text { Alta (3) }\end{array}$ & & & & \\
\hline Dif_IHDc_5 L-S y Dom (día) & $0,04 \%$ & R1 & Alta (4) & Baja (1) & & & & \\
\hline Prom_IHDv_5 & $3,52 \%$ & R4 & & & Alta (4) & Baja (1) & & \\
\hline DesvEst_IHDv_7 & 0,66 & R4 & & & & & Alta (4) & Baja (1) \\
\hline$\%$ IHDc_3 en THDc & $29,64 \%$ & $\mathrm{R} 2$ & & & & & $\begin{array}{l}\text { Media } \\
\text { Baja (2) }\end{array}$ & $\begin{array}{l}\text { Media } \\
\text { Alta (3) }\end{array}$ \\
\hline \% IHDc_5 en THDc & $89,62 \%$ & R4 & & & & & Baja (1) & Alta (4) \\
\hline Total & & & 6 & 4 & 4 & 1 & 7 & 8 \\
\hline
\end{tabular}

Fuente: Elaboración propia.

Tabla 9. Clasificación de la probabilidad para selección de clientes en barraje 2 de 13.2 kV de la S/E2.

\begin{tabular}{|c|c|c|c|c|c|c|c|c|}
\hline \multirow{2}{*}{ Característica } & \multirow{2}{*}{ Valor } & \multirow{2}{*}{ Rango } & \multicolumn{2}{|c|}{ Serv_Contratado } & \multicolumn{2}{|c|}{ Tarifa } & \multicolumn{2}{|c|}{ Zona } \\
\hline & & & $1 \phi$ & $3 \phi$ & Res & Ind & Rural & Urb \\
\hline \% IHDv_7 en THDv & $34,44 \%$ & $\mathrm{R} 2$ & $\begin{array}{c}\text { Media } \\
\text { Baja (2) }\end{array}$ & $\begin{array}{c}\text { Media } \\
\text { Alta (3) }\end{array}$ & & & & \\
\hline Dif_IHDc_5 L-S y Dom (día) & $0,35 \%$ & R1 & Alta (4) & Baja (1) & & & & \\
\hline Prom_IHDv_5 & $2,88 \%$ & R3 & & & $\begin{array}{l}\text { Media } \\
\text { Alta (3) }\end{array}$ & $\begin{array}{c}\text { Media } \\
\text { Baja (2) }\end{array}$ & & \\
\hline DesvEst_IHDv_7 & 0,59 & R4 & & & & & Alta (4) & Baja (1) \\
\hline$\%$ IHDc_3 en THDc & $78,42 \%$ & R4 & & & & & Alta (4) & Ваја (1) \\
\hline \% IHDc_5 en THDc & $44,28 \%$ & $\mathrm{R} 2$ & & & & & $\begin{array}{c}\text { Media } \\
\text { Alta (3) }\end{array}$ & $\begin{array}{c}\text { Media } \\
\text { Baja (2) }\end{array}$ \\
\hline Total & & & 6 & 4 & 3 & 2 & 11 & 4 \\
\hline
\end{tabular}

Fuente: Elaboración propia. 
En las figuras 13,14 y 15 se observan los cambios en la distorsión armónica al ocurrir un evento en la red de distribución, y con ayuda de la puntuación Z (Triola, 2009) se hace la comparación requerida y se puede dar una clasificación al impacto en la distorsión armónica; para esto se toman los eventos en los barrajes escogidos, determinando el detalle como día de la semana y rango de duración; estas variables son la que permiten realizar la comparación del comportamiento armónico durante estos eventos y las mediciones en los mismos rangos de tiempo en un histórico de seis meses de mediciones. De este modo las variaciones negativas en las gráficas denotan una disminución importante en la distorsión y los clientes afectados durante estos eventos tienen gran posibilidad de ser quienes afectan la calidad de la potencia; por esto se resaltan dentro de la primera clasificación efectuada mediante el modelo por correlación.

En la tabla 10 se observan los eventos con afectación a clientes conectados en las tres barras de estudio, de los cuales se determinan como relevantes los eventos en donde la puntuación Z es inferior a menos dos (-2), por lo que siendo consecuentes con el teorema de Chebyshev, el valor de la distorsión armónica en el momento de la falla se encuentra dentro de $25 \%$ de los valores menos probables y alejados más de dos desviaciones estándar de la media.

Con base en lo anterior, de los eventos analizados el único que permite determinar a los clientes afectados como posibles fuentes de distorsión armónica es el ocurrido sobre la barra 1 de la subestación S/E2 cuyo número de evento es CD04771562 en su primera etapa (CD04771562-1).

Tabla 10. Eventos con afectación de clientes en las barras seleccionadas.

\begin{tabular}{clccc}
\hline Barra & Evento-Etapa & $\begin{array}{c}\text { CCDD } \\
\text { Afectados }\end{array}$ & Fecha Evento & $\begin{array}{c}\text { Duración } \\
\text { [min] }\end{array}$ \\
\hline B12 & $5440575-1$ & 1 & $23 / 06 / 201307: 40$ & 450 \\
\hline B12 & $5593699-1$ & 1 & $28 / 07 / 2013 ~ 09: 23$ & 169 \\
\hline B12 & CD04737408-1 & 6 & $08 / 06 / 2013 ~ 16: 02$ & 76 \\
\hline B12 & CD04743790-3 & 5 & $15 / 07 / 2013 ~ 12: 21$ & 1973 \\
\hline B1 & $5996991-1$ & 1 & $17 / 11 / 201308: 52$ & 20 \\
\hline B1 & $5996991-3$ & 1 & $18 / 11 / 201310: 07$ & 31 \\
\hline B1 & CD04771562-1 & 15 & $02 / 11 / 201309: 00$ & $\underline{81}$ \\
\hline B1 & CD04771562-2 & 28 & $02 / 11 / 201310: 20$ & 57 \\
\hline B1 & CD04771562-3 & 17 & $02 / 11 / 201311: 18$ & 3229 \\
\hline B1 & CD04784508-2 & 154 & $29 / 12 / 201309: 24$ & 37 \\
\hline B2 & CD04774550-1 & 34 & $13 / 11 / 201314: 24$ & 3446 \\
\hline B2 & CD04774550-3 & 34 & $17 / 11 / 201311: 50$ & 346 \\
\hline
\end{tabular}

Fuente: Elaboración propia. 


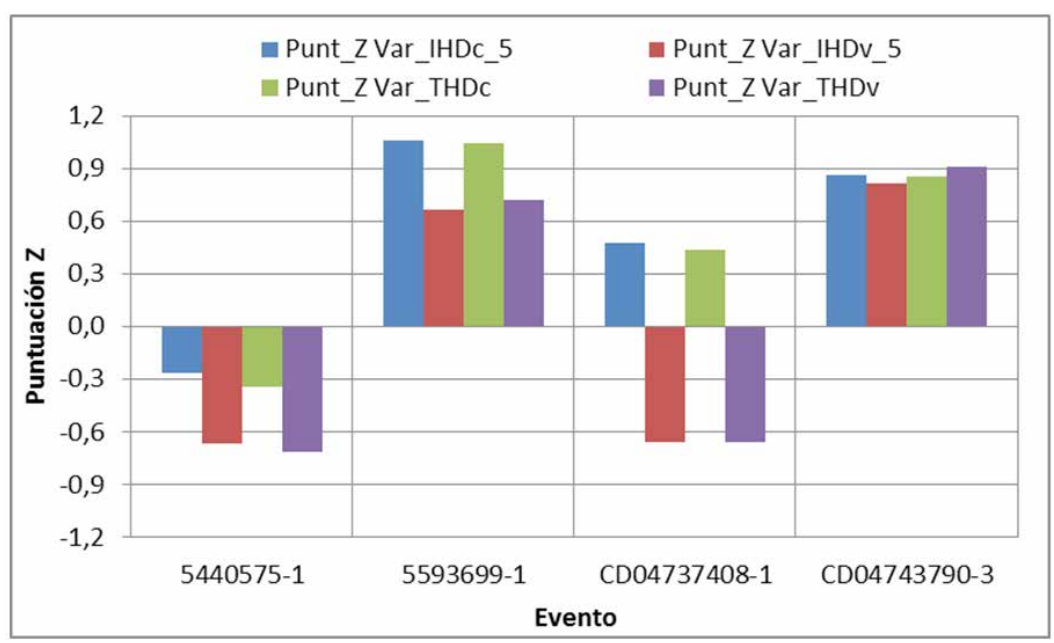

Figura 13. Puntuación $Z$ de los valores de distorsión armónica durante los eventos ocurridos en la barra 12 de 34,5 $\mathrm{kV}$ de la S/E1.

Fuente: Elaboración propia.

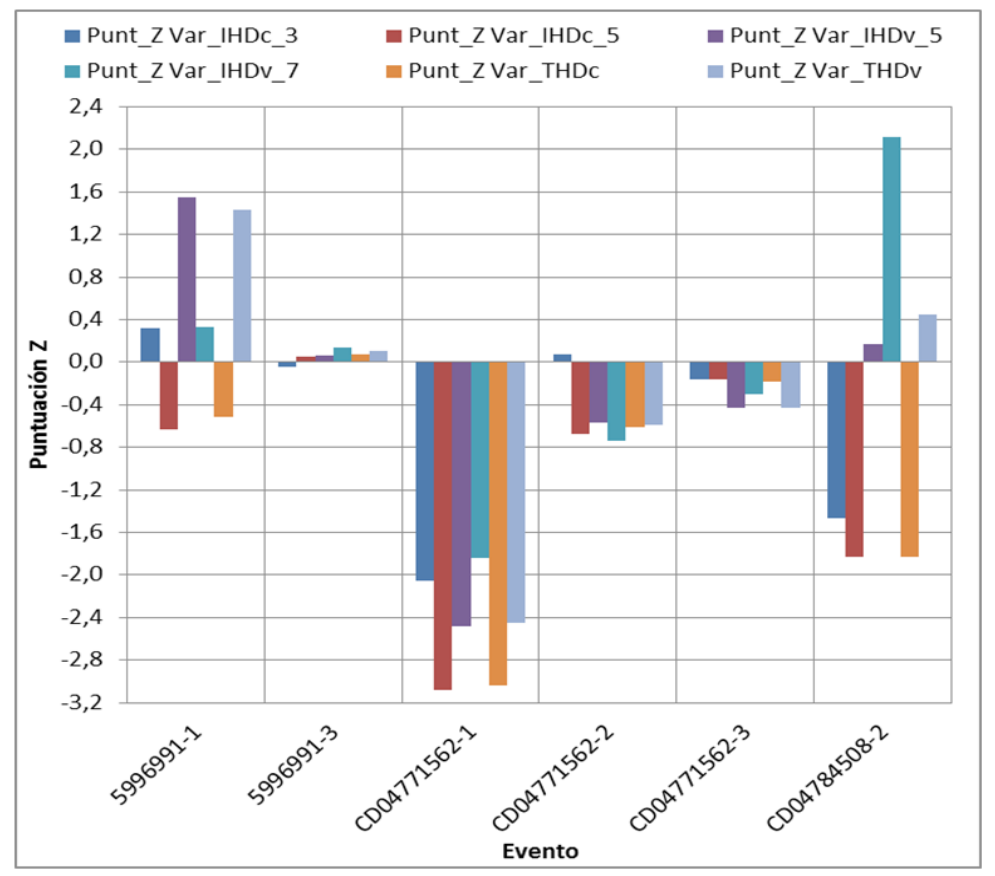

Figura 14. Puntuación $Z$ de los valores de distorsión armónica durante los eventos ocurridos en la barra 1 de 13,2 kV de la S/E2.

Fuente: Elaboración propia. 


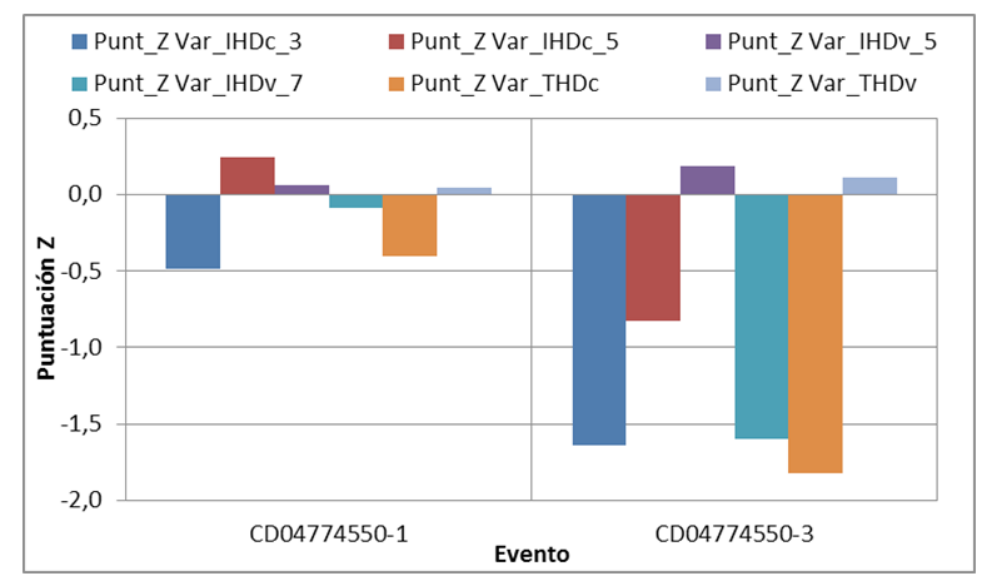

Figura 15. Puntuación Z de los valores de distorsión armónica durante los eventos ocurridos en la barra 2 de 13,2 kV de la S/E2.

Fuente: Elaboración propia.

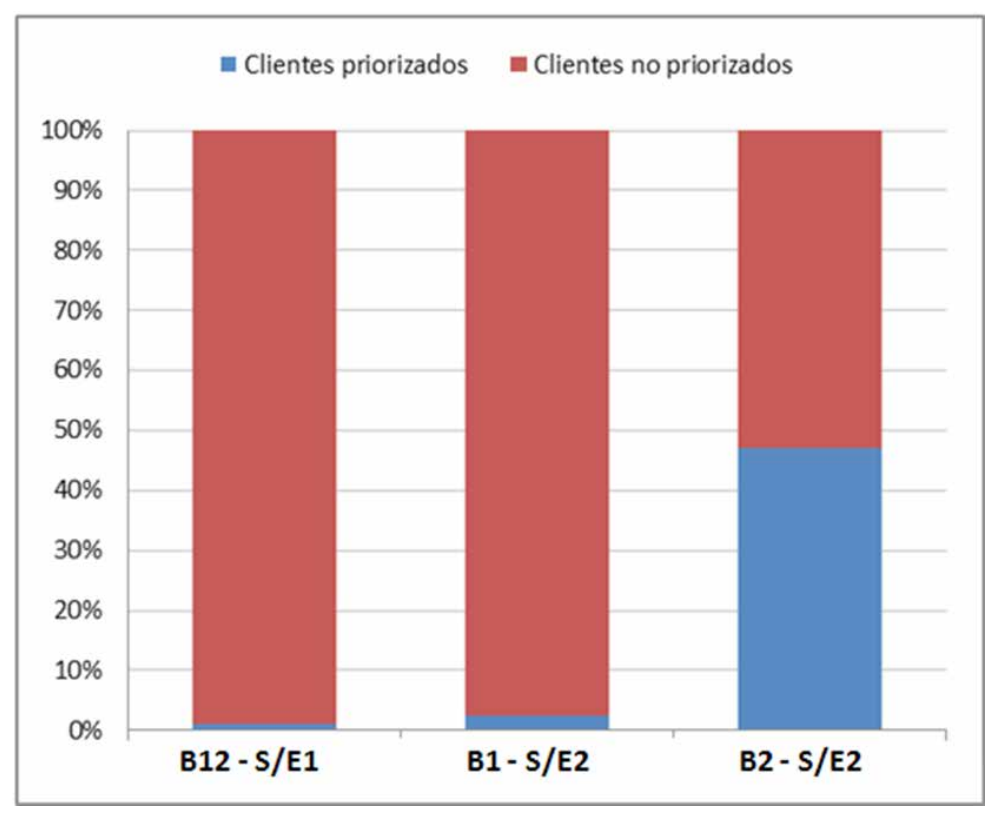

Figura 16. Porcentaje de clientes priorizados para realizar la medida de calidad de potencia en PCC para las barras seleccionadas.

Fuente: Elaboración propia.

Con el análisis de la puntuación Z calculada para cada una de las fallas se puede proceder a realizar el cruce de los centros de distribución asociados a la falla representativa, para de estos escoger los clientes que tengan las características relacionadas en la tabla 8 y así poder cerrar el proceso con una priorización precisa que permita obtener la eficiencia en las medidas en PCC para encontrar cargas generadoras de distorsión armónica. 


\section{Análisis del modelo}

Al analizar y ejecutar el modelo propuesto se obtuvo para la barra 12 de $34,5 \mathrm{kV}$ de la S/E1, la población sobre la cual se puede priorizar la medición de calidad de potencia en PCC es de 1 cliente de 107 posibles; asimismo para la barra 1 de 13,2 kV de la S/E2 se priorizará la medida a 54 de los 2211 clientes conectados en dicha barra; por último, en la barra 2 de 13,2 kV en 1932 clientes de 4097 posibles es conveniente iniciar la medida. Lo anterior se refleja gráficamente en la figura 16.

\section{CONCLUSIONES}

El método de correlación es una muy buena herramienta para focalizar variables y permitir interpretar sus efectos, relaciones y comportamiento ya que permite de manera eficiente evaluar la relación entre dos o más variables sin importar la escala de cada rango de datos por analizar.

Del análisis de las correlaciones relacionadas con el servicio contratado de los clientes observado en la figura 7 se concluye que al aumentar los clientes monofásicos y disminuir los trifásicos aumenta el porcentaje del armónico de tensión 7 y la diferencia del porcentaje del armónico de corriente 5 calculado de lunes a sábado, y el calculado los domingos disminuye.

Al aplicar el análisis mediante puntuación Z de los eventos con afectación de clientes, se puede reconocer fácilmente si las cargas desconectadas en cada una de las etapas de los eventos generan una disminución importante en la distorsión armónica, permitiendo sesgar aún más la cantidad de clientes que serán priorizados para la medida. En caso de requerirse se puede aumentar el rango de tiempo durante el cual se evalúen los eventos para tener mayor cantidad de probabilidad de encontrar una carga que al desconectarse disminuya de manera relevante la distorsión armónica.
Mediante la aplicación del modelo se logró dar prioridad en la medida en PCC a 0,93\% del total de los clientes conectados a la barra de 34,5 kV (barra 12 de la S/E1), a 2,44\% del total de los clientes conectados a la barra 1 de la S/E2 y a $47,16 \%$ de los clientes conectados a la barra 2 de la S/E2.

Para el OR la aplicación del modelo propuesto mejora sustancialmente su imagen corporativa y optimiza la utilización de recursos para la ubicación de usuarios perturbadores de la calidad de potencia por distorsión armónica, garantizando la ubicación estratégica de los equipos de medida, reduciendo sustancialmente los tiempos de ubicación y permitiendo tener respuestas oportunas tanto a los entes regulatorios como a los propios usuarios del sistema.

\section{FINANCIAMIENTO}

Universidad Distrital Francisco José de Caldas

\section{REFERENCIAS}

Comisión de Regulación de Energía y Gas (2005). CREG-024.

Comisión de Regulación de Energía y Gas (2007). CREG-017.

Dugan, M.; Santoso, \& Wayne (junio de 2013). www. digitalengineeringlibrary.com. Recuperado el 21 de marzo de 2014.

ICONTEC (3 de abril de 2002). Procedimiento de muestreo para inspecciones de atributos. Colombia.

IEEE (1992). Recommeded Practices and requirements for harmonics control in electrical power systems.

Pereira, U. T. (2010). Diagnóstico de la calidad de potencia en la actividad de distribución de energía eléctrica. Pereira.

Triola, M. (2009). Estadística. Pearson Educación.

\section{(c) $(1) \Theta \Theta$}


